\title{
UNIFORMIZAÇÃO DA COR DA MADEIRA JOVEM DE TECA PELA TERMORRETIFICAÇÃO ${ }^{1}$
}

Juliana de Oliveira Lopes², Rosilei Aparecida Garcia ${ }^{3}$, Alexandre Miguel do Nascimento ${ }^{3}$ e João Vicente de Figueiredo Latorraca ${ }^{3}$

\begin{abstract}
RESUMO - O objetivo deste estudo foi avaliar a uniformidade da cor da madeira de teca (peças contendo cerne e alburno) após a aplicação de tratamentos termorretificadores. A madeira utilizada foi oriunda de árvores jovens (12 anos) provenientes de plantios de diferentes espaçamentos. Amostras de madeira com 150 x 75 x $20 \mathrm{~mm}$ foram termorretificadas a 180 e $200^{\circ} \mathrm{C}$, durante $2 \mathrm{~h} 30$. As análises colorimétricas foram realizadas com o auxílio de um espectrofotômetro no espaço CIE-L*a*b*. Os tratamentos termorretificadores proporcionaram maior uniformidade às peças de madeira de teca, sendo o tratamento a $200{ }^{\circ} \mathrm{C}$ o mais efetivo, o que demonstra o potencial da termorretificação para a obtenção de produtos de madeira de maior valor agregado. A cor da madeira termorretificada a $180^{\circ} \mathrm{C}$ apresentou maior uniformidade no espaçamento menos adensado, entretanto ele não apresentou efeito na uniformidade da cor da madeira termorretificada a $200^{\circ} \mathrm{C}$.
\end{abstract}

Palavras-chave: Espaço CIE-L*a*b*; Tectona grandis; Tratamentos termorretificadores.

\section{COLOR UNIFORMIZATION OF THE YOUNG TEAK WOOD BY HEAT TREATMENT}

\begin{abstract}
The purpose of this study was to evaluate the uniformity of the color of the teak wood (pieces containing heartwood and sapwood) after application of heat treatments. The wood was originated from young trees (12 years old) from plantations of different spacings. Wood samples of $150 \times 75 \times 20 \mathrm{~mm}$ were heat treated at 180 and $200^{\circ} \mathrm{C}$ during 2:30 hours. Color analysis were performed by using a spectrophotometer in the CIE-L*a*b* space. Heat treatments provided greater uniformity to the teak wood pieces and the treatment at $200^{\circ} \mathrm{C}$ was the most effective, which demonstrates the potential of heat treatment to obtain wood products of higher added value. The color of the wood heat-treated at $180^{\circ} \mathrm{C}$ had higher uniformity in the least dense spacing; however, it had no effect on color uniformity of the wood heat-treated at $200{ }^{\circ} \mathrm{C}$.
\end{abstract}

Keywords: CIE- $L^{*} a * b *$ space; Tectona grandis; Heat treatments.

\section{INTRODUÇÃO}

A teca (Tectona grandis L. f.) é uma espécie arbórea da família Verbenaceae e ocorre naturalmente no Centro e Sul da Índia, em Myanmar, no Norte da Tailândia e em Laos (TSUKAMOTO FILHO et al., 2003). Tem sido identificada como uma das espécies de maior potencial para estabelecer plantações de madeira tropical de alta qualidade sob o manejo florestal sustentável (KEOGH, 1996). Essa madeira é valorizada no mercado internacional, apresentando preços mais elevados do que a madeira de mogno (Swietenia macrophylla King) (ROCHA et al., 2011).

A madeira de teca é altamente valorizada pela indústria madeireira para produção de madeira serrada e laminados em razão das suas excelentes propriedades físicas e mecânicas, bem como ao seu agradável aspecto estético (KJAER et al., 1999; SANWO, 1987).

\footnotetext{
${ }^{1}$ Recebido em 26.03.2013 aceito para publicação em 30.04.2014.

${ }^{2}$ Programa de Pós-Graduação em Ciências Ambientais e Florestais, Universidade Federal Rural do Rio de Janeiro, RJ. Email: <july.rural@gmail.com>.

${ }^{3}$ Departamento de Produtos Florestais,Universidade Federal Rural do Rio de Janeiro, RJ. E-mail: < rosileigar@ufrrj.br>, <amn@ufrrj.br> e<latorraca@hotmail.com>.
} 
A espécie apresenta cerne e alburno bem distintos. A madeira do cerne é atrativa pela sua coloração amarela ou marrom-dourada e por outras propriedades desejáveis, como a durabilidade natural, a aptidão à usinagem e ao acabamento superficial e à alta estabilidade dimensional, sendo, muitas vezes, utilizada como referência entre as espécies para a classificação de madeiras tropicais. A madeira do alburno apresenta as mesmas propriedades do cerne, entretanto possui menor durabilidade, sendo necessária a utilização de tratamentos preservativos (VIEIRA et al., 2002).

Segundo Kjaer e Foster (1996), a teca se adapta facilmente a regimes de plantações, quando comparada com outras espécies tropicais de alto valor. Essa característica associada às excelentes propriedades da madeira tem incentivado seu cultivo no mundo todo. Por razões econômicas, ciclos curtos de 20-30 anos estão sendo propostos contra a rotação tradicional de 50-60 anos (BHAT; OKUYAMA, 1997). Entretanto, ciclos mais curtos implicam obtenção de peças de madeira com maior proporção de alburno, os quais apresentam características bem distintas em termos de cor e durabilidade, quando comparados ao cerne.

No Brasil, os plantios de teca também apresentam menor ciclo de corte. De acordo com a SI-FLORESTA (2009), a madeira proveniente de árvores entre 7 e 18 anos é apropriada para a indústria moveleira, construção e decoração, apresentando alta elasticidade, o que a torna adequada para desenvolver o design final dos móveis. Entretanto, a madeira apresenta coloração mais clara. A tonalidade clara e o aspecto mesclado (contraste entre cerne e alburno) dessa madeira pode apresentar aspecto negativo, já que as madeiras de teca dos países de origem apresentam maior uniformidade quanto à cor e, consequentemente, maior aceitação no mercado em relação à madeira proveniente de plantios do Brasil.

Nos últimos anos, vários trabalhos têm demonstrado as melhorias que o tratamento de termorretificação agrega às propriedades da madeira, sendo elas, principalmente, a estabilidade dimensional e a durabilidade natural (GARCIA et al., 2012; ESTEVES et al., 2007; KAMDEM et al., 2002; VIITANIEMI; JÄMSÄ, 1994). Além dessas características, o tratamento também pode causar alterações na cor da madeira original, fazendo que ela adquira cor mais escura, a qual, muitas vezes, acaba agregando maior valor ao produto final devido à maior atratividade estética.
Na Europa, o tratamento de termorretificação é utilizado para o escurecimento de madeiras claras, de baixo valor comercial, tornando-as mais próximas da cor das madeiras tropicais, as quais são mais escuras e de alto valor comercial. Esse processo é denominado “tropicalização”. Segundo Ayadi et al. (2003), a alteração da cor pelo tratamento é particularmente apreciada para madeiras de Populus sp., Pinus sp. e Betula sp., as quais apresentam cores claras.

No Sul do Brasil, a empresa TWBrasil utiliza o tratamento térmico para melhorar determinadas propriedades tecnológicas da madeira, assim como para alterar artificialmente a cor da madeira visando aumentar a comercialização de espécies que, apesar de possuírem boas propriedades, apresentam menor procura no mercado devido à sua baixa atratividade estética. No caso da teca, o tratamento é aplicado para modificar a coloração para marrom- caramelo, obtendo-se, assim, tonalidade semelhante à das madeiras nativas (RILLING, 2009).

Nesse contexto, o objetivo geral deste estudo foi avaliar o efeito de tratamentos termorretificadores na uniformidade da cor da madeira de teca (Tectona grandis L. f.) plantada em diferentes espaçamentos.

\section{MATERIAL E MÉTODOS}

\subsection{Material e tratamento de termorretificação}

Árvores de teca (Tectona grandis L. f.) com 12 anos de idade foram obtidas de um plantio localizado na área experimental do Instituto Federal de Educação, Ciência e Tecnologia do Mato Grosso, localizado em Cáceres, MT. Foram selecionadas nove árvores provenientes de três diferentes espaçamentos: 4 × $2 \mathrm{~m}$, 5 x 2 m e 6 × 2 m, sendo consideradas três árvores de cada espaçamento, das quais foram retiradas toras a partir do DAP, que representa a medida do diâmetro do tronco da árvore tomada a 1,30 m de altura. As toras foram processadas e amostras (contendo cerne e alburno) de 150 x 75 x 20 mm (comprimento x largura x espessura), obtidas. As amostras de madeira apresentaram aproximadamente $50 \%$ de cerne e $50 \%$ de alburno. O material foi seco ao ar livre e, em seguida, acondicionado em câmara climática a $20^{\circ} \mathrm{C}$ e $65 \%$ de umidade relativa até atingir o teor de umidade de equilíbrio.

A termorretificação foi realizada em um forno mufla elétrico laboratorial, da marca Linn Elektro Therm, com dimensões de 600 x 600 x 700 mm equipado com um 
sistema de controle de temperatura e tempo. Os tratamentos termorretificadores foram realizados em quatro etapas, sendo selecionadas duas temperaturas finais de termorretificação: 180 e $200{ }^{\circ} \mathrm{C}$. As quatro etapas do tratamento foram: (1) aquecimento do material até $100^{\circ} \mathrm{C}$ : período correspondente a $2 \mathrm{~h}$; (2) aumento da temperatura de $100^{\circ} \mathrm{C}$ até a temperatura final de termorretificação ( 180 e $200^{\circ} \mathrm{C}$ ): período correspondente a $30 \mathrm{~min}$; (3) tempo de tratamento na temperatura de termorretificação: 2 h e 30 min; e (4) resfriamento do material durante aproximadamente $1 \mathrm{~h}$. O ciclo total de tratamento correspondeu a $6 \mathrm{~h}$. O teor de umidade inicial das amostras foi em torno de $8-10 \%$.

Após os tratamentos termorretificadores, as amostras foram novamente acondicionadas em câmara climática a $20^{\circ} \mathrm{C}$ e $65 \%$ de umidade relativa até atingir o teor de umidade de equilíbrio.

\subsection{Análises colorimétricas}

As análises colorimétricas foram realizadas no espaço de cor CIE-L*a*b*, antes e depois dos tratamentos termorretificadores, com o auxílio do espectrofotômetro portátil CM 2600d, versão 1.41, da Konica Minolta, o qual permitiu realizar medições de cor através do contato direto entre a superfície da amostra, em pontos predeterminados, e a área de iluminação do aparelho, com diâmetro de abertura de 3 mm (SAV "smal area view”). Utilizou-se o iluminante-padrão $\mathrm{D}_{65}$, ângulo de observação de $10^{\circ}$ e luz especular incluída. Para efetuar as medições, o aparelho foi calibrado com o padrão preto e branco, e para a calibração do branco utilizou-se placa CM-A145.

Antes das medições, as superfícies das amostras foram polidas com uma sequência de lixas de granulometria de 80 e 120, a fim de eliminar as irregularidades e obter uma superfície fresca.

Os dados coletados pelo espectrofotômetro foram, então, transferidos para o software SpectraMagic ${ }^{\mathrm{TM}}$ NX (CM-S100w), versão 1.9, da Konica Minolta e, então, exportados para o software Microsoft Office EXCEL.

Para determinar a uniformidade da cor da madeira, foram obtidos os valores das coordenadas $L^{*}$ (luminosidade), a* (tonalidade vermelha) e b* (tonalidade amarela) nas amostras de madeira contendo cerne e alburno. As medições foram tomadas em posições predeterminadas a 25, 50 e $75 \%$ do comprimento da amostra (Figura 1). Esse procedimento foi feito nas madeiras não termorretificadas e nas termorretificadas. A uniformidade foi obtida através da diferença dos valores das coordenadas entre a região do cerne e a do alburno. As variações das coordenadas de cor no interior da amostra $\left(\Delta \mathrm{L}^{*}, \Delta \mathrm{a}^{*} \mathrm{e} \Delta \mathrm{b}^{*}\right)$ foram calculadas pelas seguintes equações:

$$
\begin{aligned}
& \Delta L^{*}=\frac{\left(L{ }_{6}{ }_{6}-L{ }_{1}|+| L{ }_{5}-L{ }_{2}|+| L *_{4}-L{ }_{3} \mid\right)}{3} \\
& \Delta a^{*}=\frac{\left(a_{6}^{*}-a_{1}^{*}|+| a_{5}^{*}-a_{2}^{*}|+| a_{4}^{*}-a_{3}^{*} \mid\right)}{3} \\
& \Delta b^{*}=\frac{\left(b_{{ }_{6}}^{*}-b{ }_{1}|+| b^{*}{ }_{5}-b{ }_{2}|+| b^{*}{ }_{4}-b{ }_{3} \mid\right)}{3}
\end{aligned}
$$

em que $\Delta \mathrm{L}^{*}, \Delta \mathrm{a}^{*}$ e $\Delta \mathrm{b}^{*}$ : variação média das coordenadas $\mathrm{L}^{*}$, a* e b* no interior da amostra; $L^{*}{ }_{1}$, $L^{*}{ }_{2}$ e $L^{*}{ }_{3}$ : valores de $L^{*}$ obtidos do cerne a 25, 50 e $75 \%$ do comprimento da amostra; $L{ }_{6}^{*} L_{5}{ }_{5} \mathrm{e} L_{4}^{*}$ : valores de $L^{*}$ obtidos do alburno a 25, 50 e $75 \%$ do comprimento da amostra; $a{ }_{1}^{*} a_{2}{ }_{2}$ e $a^{*}$ : valores de a* obtidos para o cerne a 25, 50 e $75 \%$ do comprimento da amostra; $a{ }_{6}, a_{5}{ }_{5}$ e $a{ }_{4}$ : valores de a* obtidos do alburno a 25, 50 e $75 \%$ do comprimento da amostra; $b{ }_{1}^{*} b{ }_{2}{ }_{2}$ e $b{ }_{3}$ : valores de $b^{*}$ obtidos do cerne a 25,50 e $75 \%$ do comprimento da amostra; $b{ }_{6}{ }_{6}{ }^{*}{ }_{5}$ e $b{ }_{4}$ : valores de b* obtidos do alburno a 25, 50 e $75 \%$ do comprimento da amostra. A Figura 2 apresenta os pontos de medição das coordenadas colorimétricas na região do cerne e do alburno.

A variação total da cor no interior da amostra ( $\left.\Delta \mathrm{E}^{*} \mathrm{ab}\right)$ foi calculada através da seguinte equação:

$$
\Delta E^{*} a b=\sqrt{(\Delta L *)^{2}+(\Delta a *)^{2}+\left(\Delta b^{*}\right)^{2}}
$$

Essa variável foi calculada de acordo com a definição dada pela Konica Minolta (1998), seguindo-se a norma CIE “Commission Internationale de L’Éclairage” (1976).

\subsection{Análises estatísticas}

Os dados obtidos de $\Delta \mathrm{L}^{*}, \Delta \mathrm{a}^{*}, \Delta \mathrm{b}^{*}$ e $\Delta \mathrm{E}^{*} \mathrm{ab}$ foram submetidos ao teste de Kolmogorov-Smirnov a 5\% de significância, para verificar a normalidade dos dados, pois através desse teste se pode determinar se duas distribuições de probabilidade subjacentes diferem uma da outra ou se uma das distribuições de 


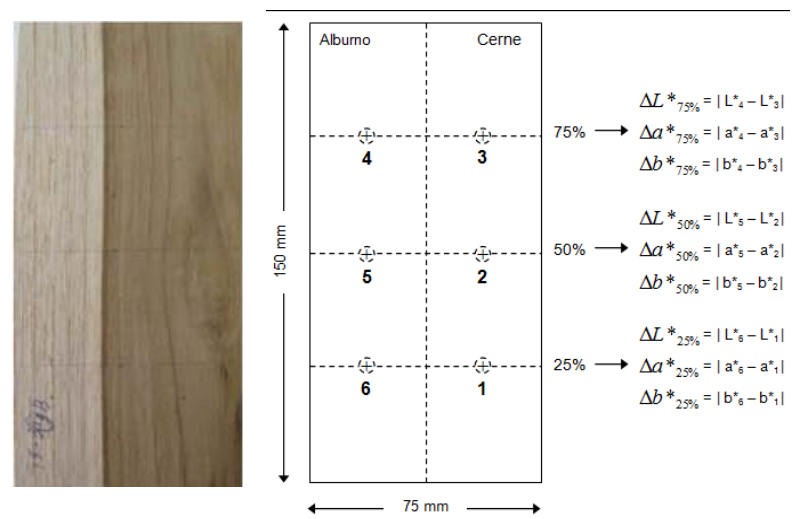

Figura 1 - Esquema dos pontos de medição das coordenadas $\mathrm{L}^{*}, \mathrm{a}^{*}$ e b*, antes e depois dos tratamentos termorretificadores, para determinação da uniformidade da cor da madeira de teca.

Figure 1 - Scheme of the measurements points of the $L^{*}$, $a^{*}$ and $b^{*}$ coordinates, before and after heat treatments to determine the color uniformity of teak wood.

probabilidade subjacentes difere da distribuição em hipótese, em qualquer dos casos com base em amostras finitas. Os dados de $\Delta \mathrm{L}^{*}, \Delta \mathrm{a}^{*}, \Delta \mathrm{b}^{*} \mathrm{e} \Delta \mathrm{E}^{*} \mathrm{ab}$ também foram submetidos ao teste de Levene, para verificar a homogeneidade das variâncias, no mesmo nível de significância, pois através dele se testa se k amostras tem a mesma variância. Alguns testes estatísticos, como a análise de variância, assumem que as variâncias são iguais dentro das categorias da variável independente ou amostras, e o teste de Levene pode ser usado para verificar essa suposição. Após a verificação desses pressupostos, as variáveis foram submetidas a uma análise de variância (ANOVA), com o auxílio do programa STATISTICA 7.0, a fim de determinar o efeito das variáveis independentes. Quando o valor F foi significativo ( $\alpha=0,05)$, aplicou-se o teste de Tukey a 5\% de significância, a fim de determinar qual contraste entre as médias apresentou diferença significativa para cada variável estudada. Através desse teste, testa-se qualquer contraste, sempre, entre duas médias de tratamentos, ou seja, não permite comparar grupos entre si. Como o teste de Tukey é, de certa forma, independente do teste F, é possível que, mesmo sendo significativo o valor de Fcalculado, não se encontrem diferenças significativas entre contrastes de médias.

As variáveis independentes consideradas foram: espaçamento de plantio (três níveis: quatro x 2 m, 5 x 2 m e 6 x 2 m) e temperatura de termorretificação (dois níveis: 180 e $200^{\circ} \mathrm{C}$ ). Esse delineamento experimental resultou em seis tratamentos com cinco repetições, resultando em um total de 30 observações. Para a madeira não termorretificada, verificou-se apenas o efeito dos espaçamentos nas variações das coordenadas de cor entre cerne e alburno.

\section{RESULTADOS}

A variação da luminosidade $\left(\Delta L^{*}\right)$ entre a madeira de cerne e de alburno não termorretificada apresentou comportamento similar nos três espaçamentos, com valores médios variando entre 13,27 e 15,84, não apresentando diferenças significativas (Tabelas 1 e 2). Entretanto, após os tratamentos termorretificadores, $\Delta \mathrm{L}^{*}$ apresentou diferenças significativas entre os espaçamentos e as temperaturas, assim como interação entre espaçamento e temperatura (Tabela 1). Nos três espaçamentos, $\Delta \mathrm{L}^{*}$ foi significativamente menor no tratamento mais agressivo $\left(200^{\circ} \mathrm{C}\right)$ quando comparado com o tratamento a $180{ }^{\circ} \mathrm{C}$. No tratamento a $180{ }^{\circ} \mathrm{C}$, $\Delta \mathrm{L}^{*}$ ainda apresentou-se alto, com diferenças significativas entre os espaçamentos de $4 \times 2$ m e 6 x 2 m, cujos valores médios foram de 14,54 e 9,43, respectivamente (Tabela 2 ). No tratamento a $200{ }^{\circ} \mathrm{C}$, os valores médios de $\Delta \mathrm{L}^{*}$ situaram-se entre 2,48 e 2,95, não havendo diferenças significativas entre os espaçamentos (Figura 2). Esses resultados indicam que as peças de madeira termorretificadas a $200{ }^{\circ} \mathrm{C}$ apresentaram maior uniformidade em termos de luminosidade. Esse efeito se deve ao fato de o alburno apresentar maior variação de luminosidade ( $\mathrm{L}^{*}$ ) após os tratamentos termorretificadores que o cerne. A Figura 4 mostra o aspecto mais uniforme das peças de madeira contendo cerne e alburno.

Para a variável $\Delta \mathrm{a}^{*}$ das madeiras não termorretificadas, as análises estatísticas indicaram diferenças significativas entre os espaçamentos de 5 x 2 m e 6 × 2 m, sendo no espaçamento de 5 x 2 m essa variação menor (Tabelas 1 e 2). Após a termorretificação, os valores de $\Delta a^{*}$ entre cerne e alburno foram menores, com valores entre 0,79 e 1,66, quando comparados com $\Delta a^{*}$ das madeiras antes da termorretificação, o que contribuiu para maior uniformidade da cor entre cerne e alburno dentro da peça.

A variável $\Delta \mathrm{b} *$ da madeira antes do tratamento (não termorretificada) apresentou comportamento semelhante nos três espaçamentos, não apresentando 
Tabela 1 - Resultados obtidos pela análise de variância (valor de F) das variáveis de cor da madeira de teca antes e depois da termorretificação.

Table 1 - Results obtained by the analysis of variance (F value) of the color variables of teak wood before and after heat treatments.

\begin{tabular}{|c|c|c|c|c|c|c|c|c|}
\hline \multirow[t]{2}{*}{ Fonte de variação } & \multirow{2}{*}{$\begin{array}{c}\text { ÄL* } \\
\text { NT }\end{array}$} & \multicolumn{3}{|c|}{ Äa* } & \multirow{2}{*}{$\begin{array}{c}\mathrm{Äb}^{*} \\
\mathrm{NT}\end{array}$} & \multicolumn{3}{|c|}{$\ddot{A} E^{*} \mathrm{ab}$} \\
\hline & & $\mathrm{T}$ & NT & $\mathrm{T}$ & & $\mathrm{T}$ & NT & $\mathrm{T}$ \\
\hline Espaçamento & $2,77^{\mathrm{NS}}$ & $4,97 *$ & $418,15 *$ & $2,25^{\mathrm{NS}}$ & $2,21^{\mathrm{NS}}$ & $6,08 *$ & $1,75^{\mathrm{NS}}$ & $6,70^{*}$ \\
\hline Temperatura & - & $167,18 *$ & - & $2,41^{\mathrm{NS}}$ & - & $4,90 *$ & - & $103,87 *$ \\
\hline Espaçamento x Temperatura & - & $3,54 *$ & - & $1,00^{\mathrm{NS}}$ & - & $0,68^{\mathrm{NS}}$ & - & $0,23^{\mathrm{NS}}$ \\
\hline
\end{tabular}

$\Delta \mathrm{L}^{*}, \Delta \mathrm{a}^{*}$ e $\Delta \mathrm{b}^{*}$ : variação das coordenadas $\mathrm{L}^{*}$, a* e b* no interior da amostra. ÄE*ab: variação total da cor no interior da amostra. NT: madeira não termorretificada. T: madeira termorretificada. * Significativo a 95\% de probabilidade. ${ }^{\text {NS }}$ Não significativo.

Tabela 2 - Estatística descritiva (média e desvio-padrão) das variáveis de cor da madeira de teca antes e depois da termorretificação. Table 2 - Descriptive statistics (means and standard deviation) of the color variables of teak wood before and after heat treatments.

\begin{tabular}{|c|c|c|c|c|c|}
\hline \multirow[b]{2}{*}{ Espaçamento (m) } & \multicolumn{5}{|c|}{ Madeira não termorretificada } \\
\hline & \multicolumn{2}{|c|}{$\Delta \mathrm{L} *$} & $\Delta \mathrm{a} *$ & $\Delta \mathrm{b}^{*}$ & $\Delta \mathrm{E}^{*} \mathrm{ab}$ \\
\hline $4 \times 2$ & 14,97 & $3,96)$ & $5,73 \mathrm{ab}(2,74)$ & $1,15 \mathrm{a}(1,17)$ & $16,46 \mathrm{a}(3,65)$ \\
\hline $5 \times 2$ & 15,84 & $3,94)$ & $3,95 \mathrm{a}(2,82)$ & $1,38 \mathrm{a}(1,05)$ & $16,71 \mathrm{a}(3,64)$ \\
\hline \multirow[t]{3}{*}{$6 \times 2$} & 13,27 & $4,89)$ & $6,01 \mathrm{~b}(2,52)$ & $0,84 \mathrm{a}(0,72)$ & $15,04 \mathrm{a}(4,03)$ \\
\hline & $\mathrm{N}$ & & $\mathrm{S}$ & NS & NS \\
\hline & \multicolumn{5}{|c|}{ Madeira termorretificada } \\
\hline Espaçamento (m) & Temperatura $\left({ }^{\circ} \mathrm{C}\right)$ & $\Delta \mathrm{L}^{*}$ & $\Delta \mathrm{a}^{*}$ & $\Delta b^{*}$ & $\Delta \mathrm{E} * \mathrm{ab}$ \\
\hline \multirow[t]{2}{*}{$4 \times 2$} & 180 & $14,64 \mathrm{c}(4,49)$ & $0,89 \mathrm{a}(0,72)$ & 4,46 ab(2,33) & $15,45 \mathrm{c}(4,71)$ \\
\hline & 200 & $2,95 \mathrm{a}(1,98)$ & $1,4 \mathrm{a}(1,48)$ & 3,81 ab $(3,46)$ & $5,21 \mathrm{a}(4,00)$ \\
\hline \multirow[t]{2}{*}{$5 \times 2$} & 180 & 12,45 bc $(4,72)$ & $1,11 \mathrm{a}(0,72)$ & $5,65 \mathrm{~b}(2,65)$ & 13,99 bc $(4,54)$ \\
\hline & 200 & $2,55 \mathrm{a}(1,73)$ & $1,66 \mathrm{a}(1,26)$ & 3,87 ab $(2,89)$ & $5,26 \mathrm{a}(3,04)$ \\
\hline \multirow[t]{3}{*}{$6 \times 2$} & 180 & $9,43 \mathrm{~b}(4,07)$ & $0,89 \mathrm{a}(0,58)$ & 3,11 ab $(1,71)$ & $10,12 \mathrm{~b}(4,12)$ \\
\hline & 200 & 2,48 a $(2,87)$ & 0,79 a $(0,86)$ & $1,99 \mathrm{a}(1,60)$ & 3,43 a $(3,16)$ \\
\hline & $\mathrm{S}$ & NS & $\mathrm{S}$ & $\mathrm{S}$ & \\
\hline
\end{tabular}

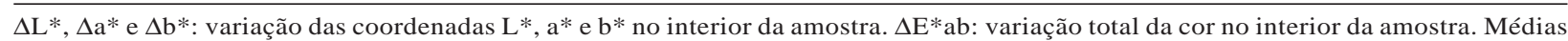
com a mesma letra não são estatisticamente diferentes entre si, segundo o teste de Tukey. NS: não significativo. S: significativo a 95\% de probabilidade.

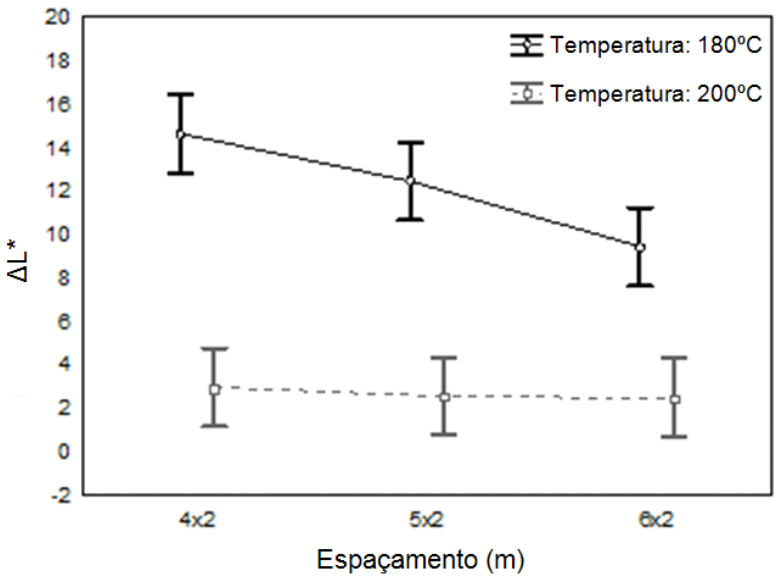

Figura 2 - Interação entre espaçamento e temperatura para variação da luminosidade $\left(\Delta \mathrm{L}^{*}\right)$ da madeira de teca após a termorretificação.

Figure 2 - Interaction between spacing and temperature for lightness variation $\left(\Delta L^{*}\right)$ of the teak wood after heat treatments. diferenças significativas entre eles (Tabela 1). Entretanto, após os tratamentos termorretificadores, ocorreu aumento da variação do pigmento amarelo ( $\left.\Delta \mathrm{b}^{*}\right)$ (Tabela 2$)$. As análises estatísticas indicaram diferenças significativas entre os espaçamentos e entre as temperaturas para $\Delta \mathrm{b}^{*}$ (Tabela 2). A variável $\Delta \mathrm{b}^{*}$ diferenciou-se entre o tratamento a $180{ }^{\circ} \mathrm{C}$ do espaçamento de $5 \times 2 \mathrm{~m}$ e o tratamento a $200{ }^{\circ} \mathrm{C}$ do espaçamento de $6 \times 2 \mathrm{~m}$, apresentando este último o menor valor $(1,99)$.

A variação total da cor entre a madeira de cerne e o alburno ( $\left.\Delta \mathrm{E}^{*} \mathrm{ab}\right)$ para a madeira não termorretificada apresentou comportamento semelhante nos três espaçamentos, não apresentando diferenças significativas entre eles (Tabelas 1 e 2).

Para as madeiras termorretificadas, a variável $\Delta \mathrm{E}^{*} \mathrm{ab}$ apresentou diferenças significativas entre os espaçamentos e as temperaturas de termorretificação 
(Tabela 1). Como pode ser observado na Tabela 2, os espaçamentos de $4 \times 2$ m e 6 × 2 m foram significativamente diferentes entre si, mas somente nas madeiras tratadas a $180{ }^{\circ} \mathrm{C}$, sendo a uniformidade entre cerne e alburno relativamente maior no espaçamento menos adensado. Isso porque, quanto menor o valor de $\Delta \mathrm{E}^{*} \mathrm{ab}$, maior a uniformidade da cor no interior da peça de madeira.

\section{DISCUSSÃO}

Os valores de $\Delta \mathrm{E}^{*}$ ab no tratamento a $200{ }^{\circ} \mathrm{C}$ (entre 3,43 e 5,21) foram significativamente inferiores aos encontrados no tratamento a $180^{\circ} \mathrm{C}$ (entre 10,12 e 15,45), em todos os espaçamentos. Isso quer dizer que o tratamento mais agressivo foi mais eficiente para uniformizar a cor das peças (Figura 3).

Os resultados encontrados para $\Delta \mathrm{E}^{*} \mathrm{ab}$ no tratamento a $200{ }^{\circ} \mathrm{C}$ corroboram aqueles encontrados por Esteves (2007), em que a cor da madeira de Pinus pinaster após o tratamento menos agressivo foi menos uniforme devido ao maior contraste entre lenho inicial e lenho tardio, sendo a cor das amostras dependente da razão entre os lenhos inicial/tardio na superfície da amostra.

O tratamento termorretificador menos agressivo $\left(180^{\circ} \mathrm{C}\right)$, apesar de não atingir a perfeita uniformidade da cor no interior da peça, proporcionou ao alburno coloração mais escura, tornando o contraste entre cerne e alburno menos evidente em relação à madeira não
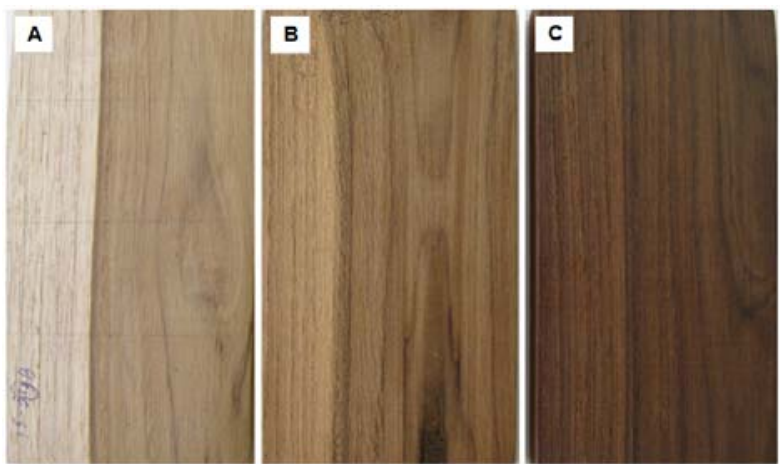

Figura 3 - Uniformidade da cor da madeira de teca, contendo cerne e alburno após os tratamentos termorretificadores. (A) Madeira original. (B) Madeira termorretificada a $180{ }^{\circ} \mathrm{C}$. (C) Madeira termorretificada a $200{ }^{\circ} \mathrm{C}$.

Figure 3-Color uniformity of the teak wood, containing heartwood and sapwood after heat treatments. (A) Original wood. (B) Wood heat-treated at $180^{\circ} \mathrm{C}$. (C) Wood heat-treated at $200{ }^{\circ} \mathrm{C}$. tratada. Portanto, este pode ser um tratamento viável para atender aos consumidores que apreciem a madeira de teca com certa uniformidade de cor, mas não tão escuras.

A alteração da cor original pode agregar maior valor ao produto final. Em países da Europa e América do Norte, o tratamento tem sido aplicado em madeiras de cores claras e de menor valor comercial, como Pinus spp. e Picea spp., visando à obtenção de produtos de maior valor agregado. De acordo com Bekhta e Niemz (2003), Mitsui et al. (2001) e Mitsui (2006), a madeira termorretificada apresenta vantagem estética, já que esta adquire cor similar às das madeiras tropicais, sendo, portanto, apreciada para inúmeras aplicações.

Embora o mercado nórdico aprecie madeiras de tons claros, em muitos outros mercados são preferidas cores mais escuras, as quais estão associadas a preços mais elevados. Por exemplo, em Portugal, a madeira de Pinus sp. é escurecida para produção de móveis de alta qualidade.

Além da maior uniformidade da cor, a madeira termorretificada de teca pode apresentar melhorias em outras propriedades (ex.: maior durabilidade do alburno). Assim, a variação de cor dessas madeiras induzida pelo calor poderia melhorar o seu valor para aplicações de alta qualidade.

A importância em estudar a uniformidade da cor da madeira de teca pode se basear em outros trabalhos realizados com outras espécies em busca de melhor estética. Tolvaj et al. (2000) estudaram a alteração e uniformidade da cor da madeira de Robinia pseudoacacia, espécie extremamente heterogênea, visando chegar a uma textura com maior aceitação no mercado.

Assim como neste estudo se buscou maior uniformidade dentro da peça da madeira de teca através da termorretificação, espécies como a faia (Fagus sylvatica) também foram submetidas ao mesmo tipo de processo para escurecer e homogeneizar sua cor e torná-la mais desejável para o mercado moveleiro (TOLVAJ; MOLNÁR, 2006).

A madeira contém compostos químicos como o tanino, que torna possível não só a alteração na cor, como também a homogeneidade artificial da cor natural da madeira pela aplicação de produtos químicos (MILLER et al., 1985; MITSUI; TOLVAJ, 2005) e de tratamentos 
térmicos (AYADI et al., 2003; MITSUI, 2006). Entretanto, a termorretificação tem a vantagem de não utilizar produtos químicos nocivos ao meio ambiente, sendo, portanto, um tratamento ecologicamente mais correto.

\section{CONCLUSÕES}

Os tratamentos termorretificadores proporcionaram maior uniformidade às peças de madeira contendo cerne e alburno, sendo o tratamento a $200{ }^{\circ} \mathrm{C}$ o mais efetivo.

A cor da madeira termorretificada a $180^{\circ} \mathrm{C}$ apresentou maior uniformidade no espaçamento menos adensado, entretanto este não exibiu efeito na uniformidade da cor da madeira termorretificada a $200{ }^{\circ} \mathrm{C}$.

A termorretificação pode ser considerada um método promissor para a obtenção de produtos de maior valor agregado.

\section{REFERÊNCIAS}

AYADI, N. et al. Color stability of heat-treated wood during artificial weathering. Holz als Roh-und Werkstoff, v.61, p.221-226, 2003.

BEKHTA, P.; NIEMZ, P. Effect of high temperature on change in color, dimensional stability and mechanical properties of spruce. Holzforschung, v.57, p.539-546, 2003.

BHAT, K. M.; OKUYAMA, T. Action plan for hardwood plantation management vis-à-vis sustainability of tropical forest resources; a concept note, Unpublished Doc., 1997.

ESTEVES, B.; DOMINGOS, I.; PEREIRA, H. Improvement of technological quality of eucalypt wood by heat treatment in air at 170$200^{\circ} \mathrm{C}$. Forest Products Journal, v.57, n.1/ 2, p.47-52, 2007.

GARCIA, R. A. et al. Nondestructive evaluation of heat-treated Eucalyptus grandis Hill ex Maiden wood using stress wave method. Wood Science and Technology, v.46, n.1, p.41-52, 2012.

KAMDEM, D. P.; PIZZI, A.; JERMANNAUD, A. Durability of heat-treated wood. Holz als

Roh-und Werkstoff, v.60, p.1-6, 2002.

KEOGH, R. M. Does teak have a future in tropical America? Unasylva, v.31, n.126, p.13-19, 1996.
KJAER, E. D.; FOSTER, G. S. The economics of tree improvement of teak (Tectona grandis L.). Humlebaek: DANIDA Forest Seed Centrev, 1996. (Moshi, 34)

KJAER, E. D.; KAJORNSRICHON, S.; LAURIDSEN, E. B. Heartwood, calcium and silica content in five provenances of teak (Tectona grandis). Silvae Genetica, v.48, p.1-3, 1999.

\section{KONICA MINOLTA SENSING Inc. Comunicação precisa da cor. 1998. 57p.}

MILLER, R. B.; QUIRK, J. T.; CHRISTENSEN, D. J. Identifying white oak logs with sodium nitrite. Forest Products Journal, v.35, n.2, p.33-38, 1985.

MITSUI, K. Changes in colour of spruce by repetitive treatment of light-irradiation and heat treatment. Holz als Roh-und Werkstoff, v.64, p.243-244, 2006.

MITSUI, K. et al. Changes in the properties of light-irradiated wood with heat treatment: Part 1. Effect of treatment conditions on the change in color. Holzforschung, v.55, p.601-605, 2001.

MITSUI, K.; TOLVAJ, L. Color changes in acetylated wood by the combined treatment by light and heat. Holz als Roh-und Werkstoff, v.63, n.5, p.392-393, 2005.

RILLING, E. A. G. Madeira modificada com calor - TMT (Thermally Modified Timber). TWBrazil, 2009. 7p.

ROCHA, R. B. et al. Caracterização de fatores que afetam a germinação de teca (Tectona grandis): temperatura e escarificação. Revista Árvore, v.35, n.2, p.205-212, 2011.

SANWO, S. K. The characteristics of the crownformed and stem-formed wood in plantation grown teak (Tectona grandis L. f.) in Nigeria. Journal of the Institute of Wood Science, v.11, n.1, p.85-88, 1987.

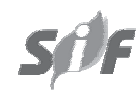

Revista Árvore, Viçosa-MG, v.38, n.3, p.561-568, 2014 
SI-FLORESTA. Sistema de Informações Florestais. Teca jovem, a nova sensação do mercado. Disponível em: http://

www.ciflorestas.com.br/conteudo.php?id=833, Acessado em: 20 de abr. de 2012.

TOLVAJ, L.; HORVÁTH-SZOVÁTI, E.; SAFAR, C. Colour modification of black locust by steaming. Wood Research, v.45, n.2, p.25-32, 2000.

TOLVAJ, L.; MOLNÁR, S. Colour homogenization of hardwood species by steaming. Acta

Silvatica et Lignaria Hungarica, v.2, p.105-112, 2006.
TSUKAMOTO FILHO, A. A. et al. Análise econômica de um plantio de teca submetido a desbastes. Revista Árvore, v.27, n.4, p.487-494, 2003.

VIEIRA, A. H. et al. Aspectos silviculturais da teca em Rondônia. Porto Velho: Embrapa CPAF - Ministério da Agricultura, Pecuária e Abastecimento, 2002. p.8. (Documentos, 68)

VIITANIEMI, P.; JÄMSÄ, S. Modification of wood with heat treatment. VTT Research Report Publications 814, Espoo, Finland, 1994. 
ERRATA - EDIÇÃO V. 38, N. 3 de 2014

\section{UNIFORMIZAÇÃO DA COR DA MADEIRA JOVEM DE TECA PELA TERMORRETIFICAÇÃO}

Pág 563, coluna 1, parágrafo 6, linha 7: onde se lê "Figura 2",deve-se ler "Figura 1";

Pág 564, coluna 2, parágrafo 2, linha 19: onde se lê "Figura 3", deve-se ler "Figura 2";

Pág 566, coluna 1, parágrafo 2, linha 6: onde se lê "Figura 4", deve-se ler "Figura 3".

Página 565, Tabela 1, onde se lê:
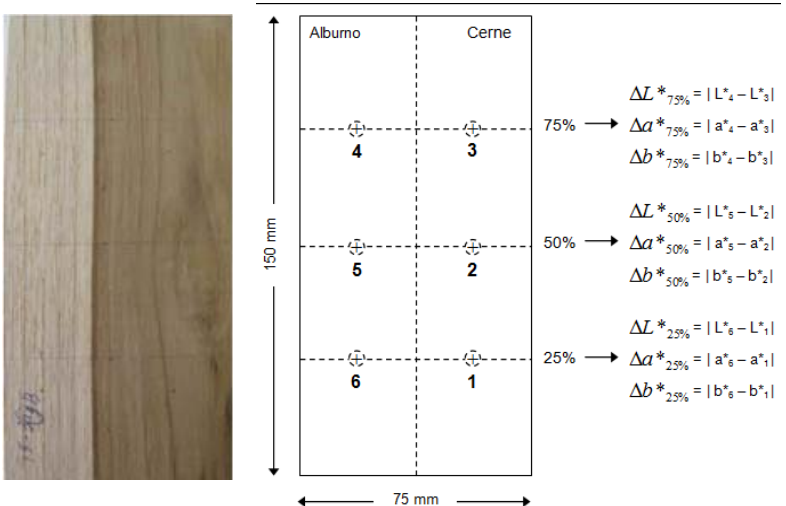

Figura 2 - Esquema dos pontos de medição das coordenadas $\mathrm{L}^{*}, \mathrm{a}^{*}$ e b*, antes e depois dos tratamentos termorretificadores, para determinação da uniformidade da cor da madeira de teca.

Figure 2 -Scheme of the measurements points of the $L^{*}$, $a^{*}$ and $b^{*}$ coordinates, before and after heat treatments to determine the color uniformity of teak wood.

Página 564, Tabela 1, onde se lê:

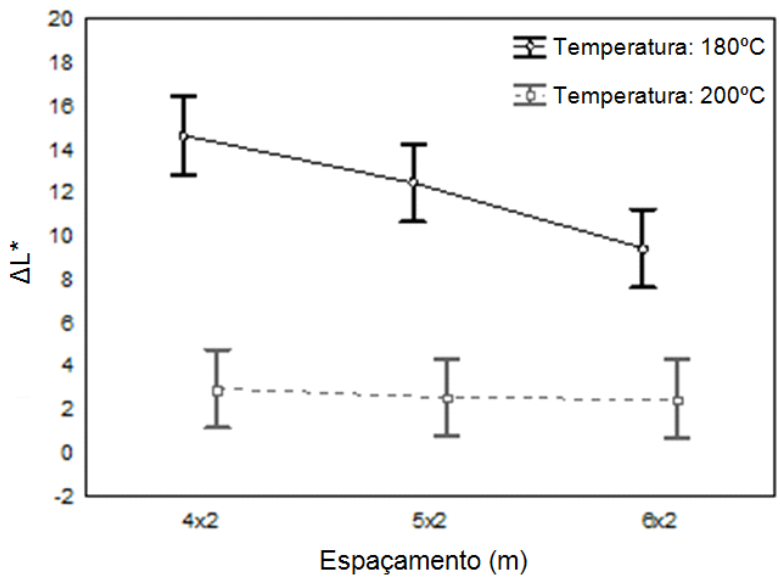

Figura 3 - Interação entre espaçamento e temperatura para variação da luminosidade $\left(\Delta \mathrm{L}^{*}\right)$ da madeira de teca após a termorretificação.

Figure 3 - Interaction between spacing and temperature for lightness variation $\left(\Delta L^{*}\right)$ of the teak wood after heat treatments. deve-se ler :
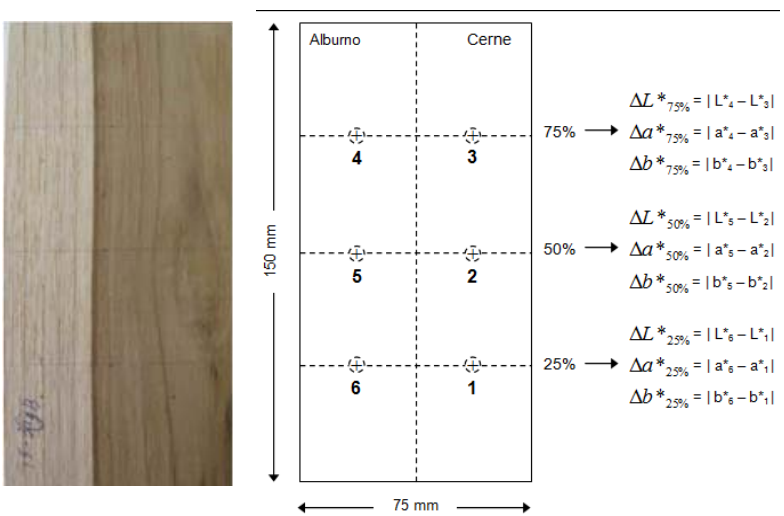

Figura 1 - Esquema dos pontos de medição das coordenadas $\mathrm{L}^{*}, \mathrm{a}^{*}$ e b*, antes e depois dos tratamentos termorretificadores, para determinação da uniformidade da cor da madeira de teca.

Figure 1 -Scheme of the measurements points of the $L^{*}$, $a^{*}$ and $b^{*}$ coordinates, before and after heat treatments to determine the color uniformity of teak wood.

deve-se ler :

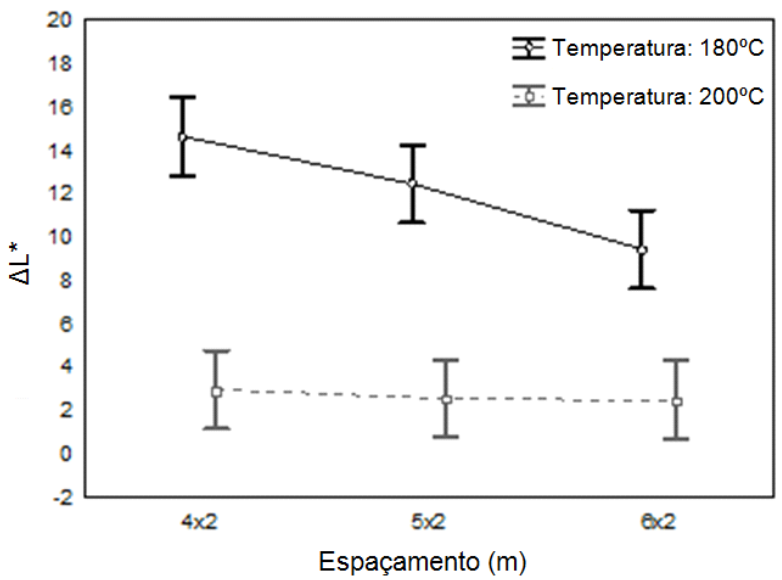

Figura 2 - Interação entre espaçamento e temperatura para variação da luminosidade $\left(\Delta \mathrm{L}^{*}\right)$ da madeira de teca após a termorretificação.

Figure 2 - Interaction between spacing and temperature for lightness variation $\left(\Delta L^{*}\right)$ of the teak wood after heat treatments.

Revista Árvore, Viçosa-MG, v.38, n.3, p.561-568, 2014 
Página 565, Tabela 2, onde se lê:

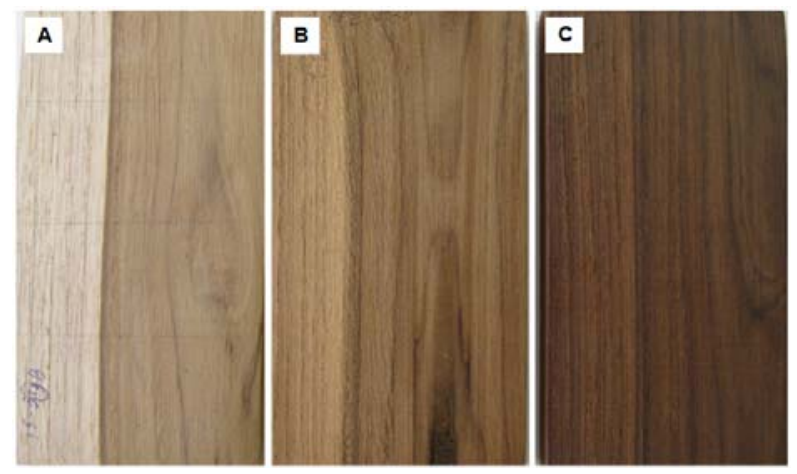

Figura 4 - Uniformidade da cor da madeira de teca, contendo cerne e alburno após os tratamentos termorretificadores. (A) Madeira original. (B) Madeira termorretificada a $180{ }^{\circ} \mathrm{C}$. (C) Madeira termorretificada a $200{ }^{\circ} \mathrm{C}$.

Figure 4-Color uniformity of the teak wood, containing heartwood and sapwood after heat treatments. (A) Original wood. (B) Wood heat-treated at $180^{\circ} \mathrm{C}$. (C) Wood heat-treated at $200^{\circ} \mathrm{C}$. deve-se ler :
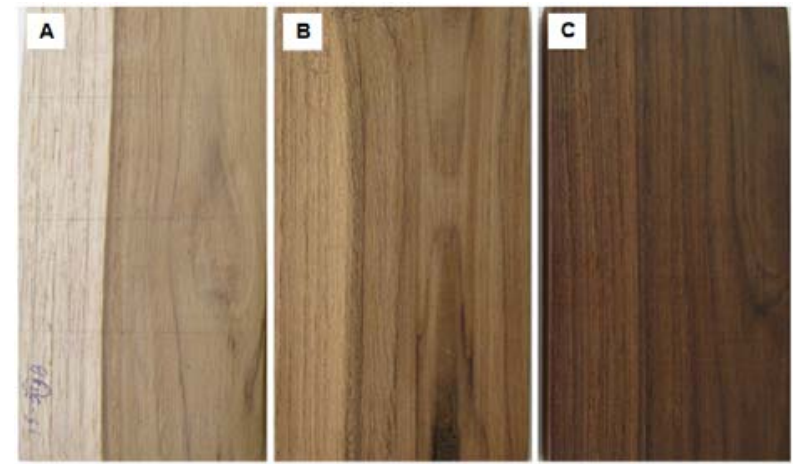

Figura 3 - Uniformidade da cor da madeira de teca, contendo cerne e alburno após os tratamentos termorretificadores. (A) Madeira original. (B) Madeira termorretificada a $180{ }^{\circ} \mathrm{C}$. (C) Madeira termorretificada a $200{ }^{\circ} \mathrm{C}$.

Figure 3 - Color uniformity of the teak wood, containing heartwood and sapwood after heat treatments. (A) Original wood. (B) Wood heat-treated at $180^{\circ} \mathrm{C}$. (C) Wood heat-treated at $200^{\circ} \mathrm{C}$. 